\title{
Some Thermodynamic Properties of the Systems Polybutadiene-Benzene and Polyisobutene-Benzene*
}

\author{
R. S. Jessup
}

\begin{abstract}
Experimental data are reported on densities at $26.9^{\circ} \mathrm{C}$ of solutions of polyisobutene in benzene covering the composition range from 0 to 10 percent by weight of polyisobutene, and on relative vapor pressures of solutions of polybutadiene and of polyisobutene in benzene covering the range from 0 to 50 percent of benzene. Values of apparent specific volume of polyisobutene in benzene were calculated from the densities of solvent and solutions. The results of the vapor pressure measurements were compared with the Flory-Huggins equation. The deviations from this equation for the polysiobutene-benzene system are much greater than for the polybutadiene-benzene system. The following equations represent the activities of the solvent for the polybutadiene-benzene and polysiobutene-benzene systems, respectively.

$$
\begin{aligned}
& \ln a_{1}=\ln v_{1}+v_{2}+0.27 v_{2}^{2}+0.06 v_{2}^{3}, \\
& \ln a_{1}=\ln v_{1}+\left(1-\frac{1}{m}\right) v_{2}+0.50 v_{2}^{2}+0.388 v_{2}^{3}+0.184 v_{2}^{12},
\end{aligned}
$$

where $a_{1}$ represents activity of the solvent, $v_{1}$ and $v_{2}$ are volume fractions of solvent and polymer, respectively, and $m$ is the ratio of molal volumes of polymer and solvent.
\end{abstract}

\section{Introduction}

Accurate measurements of thermodynamic properties of polymer solutions are of interest in connection with attem.pts to interpret the properties of such solutions in terms of statistical-mechanical treatments of various models. The present paper presents results of measurements of vapor pressures in the system polybutadiene-benzene, and of vapor pressures and solution densities in the system polyisobutenebenzene. Both polybutadiene and polyisobutene are of importance as constituents of synthetic rubbers. Polyisobutene should be of particular interest in connection with theoretical investigations of polymer solutions because of the relative simplicity of the structure of the polyisobutene molecule.

\section{Materials}

\subsection{Polybutadiene}

The sample of polybutadiene used has been described in a previous paper [1], ${ }^{1}$ where it was designated as sample B. It was prepared by emulsion polymerization at $5^{\circ} \mathrm{C}$, using a commercial tertdodecyl mercaptan as the "modifier." It was purified in the Rubber Section of the Bureau by repeatedly dissolving it in benzene and then precipitating it by pouring the solution into methyl alcohol while stirring. After purification in this manner, the sample was again dissolved in benzene, a small amount (0.14 percent by weight of polymer) of phenyl-beta-naphthylamine was added as an antioxidant, the mixture was frozen, and the benzene removed by sublimation in vacuum to yield a product

*The work described in this paper was done as part of a research project sponsored by the National Science Foundation, Polymer Research Group, in connection with the Government's synthetic rubber program.

1 Figures in brackets indicate the literature references at the end of this paper. similar to foam rubber in appearance. The procedure described above removed soap, fatty acid, and stabilizer, as well as some of the low-molecular-weight polymer. After the measurements described in reference [1] the purified sample was kept in a refrigerator in an inert atmosphere.

In the present work, in order to remove traces of benzene and air, the sample was evacuated at room. temperature to a pressure of less than $10^{-4} \mathrm{~mm}$ of mercury for about a month. It was found, however, that this did not completely remove either dissolved air (or other permanent gas) or benzene from the polymer. Permanent gases were reduced to a negligible amount by repeatedly adding benzene to the polymer and then pumping it off. Benzene was reduced to a negligible amount by evacuating to a pressure of less than $10^{-4} \mathrm{~mm}$ of mercury and heating to about $95^{\circ} \mathrm{C}$. During this process most of the phenyl-beta-naphthylamine sublimed from the sample.

\subsection{Polyisobutene}

The polyisobutene sample was obtained by fractional precipitation ${ }^{2}$ from solution of a low-molecular-weight commercial polyisobutene (Vistanex). The fraction used in the measurements had a viscosity-average molecular weight ${ }^{3}$ of 76,000 . This material was used without further purification except for removal of permanent gas and solvent. Permanent gas was removed by repeated addition of benzene to the polymer followed by removal of the benzene by evacuation. The final step in removal of benzene consisted in prolonged evacuation to a pressure of less than $10^{-4} \mathrm{~mm}$ of mercury while the temperature was gradually raised to about $140^{\circ} \mathrm{C}$.

2 The fractionation of the polymer was done in the Polymer Structure Section of the Bureau.

${ }_{3}$ The molecular weight was determined by Miss H. V. Belcher of the Rheology Section of the Bureau. 
Heating and evacuation were continued until the rate of removal of benzene was reduced to a few micrograms per day.

\subsection{Benzene}

Reagent grade benzene was purified by slow fractional freezing by a method described elsewhere [2], in which freezing proceeded upward along the length of a vertical tube containing the benzene while the liquid phase was stirred. This process was repeated twice, about 25 percent of the material being rejected each time. The resulting purified material had a density at $25^{\circ} \mathrm{C}$ of $0.87334 \mathrm{~g} / \mathrm{cm}^{3}$, as compared with the value $0.87368 \mathrm{~g} / \mathrm{cm}^{3}$ reported in the literature [3a]. This material was used without further purification for measurements of densities of solutions. It seems very improbable that the purity of the solvent has any appreciable effect on the values of apparent specific volume of solute derived from measurements of densities of solvent and solution.

For use in vapor-pressure measurements a portion of the benzene was transferred by vacuum distillation into a glass supply container which had been previously heated to $150^{\circ}$ to $200^{\circ} \mathrm{C}$ while being evacuated to about $10^{-5} \mathrm{~mm}$ of mercury. Before entering the supply container the vapor passed through a drying: tube containing magnesium perchlorate followed by phosphoric anhydride. The benzene in the supply container was then frozen in liquid air several times and evacuated to a pressure of about $10^{-5} \mathrm{~mm}$ of mercury, after which the sample was heated to room temperature and about 10 percent of the benzene was removed by evacuation. A fraction of the remaining benzene was then transferred by distillation to the previously evacuated vapor-pressure apparatus, and finally about 10 percent of the benzene in the vapor-pressure apparatus was removed by evacuation. The measured vapor pressure of the benzene so treated, in the liquid state at $0^{\circ} \mathrm{C}$, agreed within 0.01 or $0.02 \mathrm{~mm}$ of mercury with an equation for the vapor pressure of the liquid based on the best available data [3b].

\section{Apparatus and Methods}

\subsection{Density Measurements}

The apparatus and methods used have been described previously [4], and only a brief description will be given here. Densities were measured with two bicapillary Pyrex pycnometers which were used previously [4], and which are of the type described by Heller and Thompson [5]. The capacity of each pycnometer was approximately $22 \mathrm{~cm}^{3}$, and the diameter of the capillaries about $1 \mathrm{~mm}$. The mass of solution in each pycnometer was determined by weighing on a semimicro balance on which readings could be repeated to about $0.02 \mathrm{mg}$. The volume of the solution in a pycnometer was determined by observing the positions of the menisci relative to those of reference marks on the capillaries to about $0.01 \mathrm{~mm}$ by means of a Wild cathetometer, while the pycnometer was immersed in a water bath whose temperature was maintained constant to about $\pm 0.002^{\circ} \mathrm{C}$. The observations were made through a plate glass window which formed one wall of the bath. Temperature was measured to $0.001^{\circ} \mathrm{C}$ by means of a platinum resistance thermometer and a Mueller bridge. The pycnometers were calibrated by weighing when empty and when filled with distilled water at temperatures near $27^{\circ} \mathrm{C}$. All weights were corrected for air buoyancy, and for the masses of air and vapor above the liquid menisci in the capillaries.

In the previous work [4] the pycnometers were closed by glass caps fitted to the tops of the capillaries by standard-taper ground-glass joints. These could not be made to fit well enough to prevent loss of a small amount of solvent by evaporation. Also, because when the pycnometers were immersed in the water bath the caps projected out into the atmosphere which was at a lower temperature than the bath, it was suspected that some of the solvent in the capillaries might evaporate and condense in the caps. In the present work the glass caps were replaced by metal caps of the type shown in figure 1 , in which a Monel metal cone is held by a brass nut against a brass sleeve, which in turn is soft-soldered to the top of the Pyrex capillary. This type of cap can be closed tightly enough to prevent any loss of solvent. It was found that when the pycnometers were weighed with the caps not tightened, subsequent tightening of one of the caps caused no appreciable change in weight. If a cap had once been tightened, then the subsequent removal of the nut apparently removed enough metal by abrasion to cause an appreciable change in weight. The procedure in filling the pycnometers and tightening the caps was such as to avoid such changes in weight. The pycnometers were first weighed empty with the nuts loosely in place. They were then filled with solution in the manner described previously [4] and placed in the thermoregulated bath with the nuts loosely in place and with the lower part of the caps, i. e., the brass sleeves, in contact with the water in the bath. After allowing sufficient time for the attain-

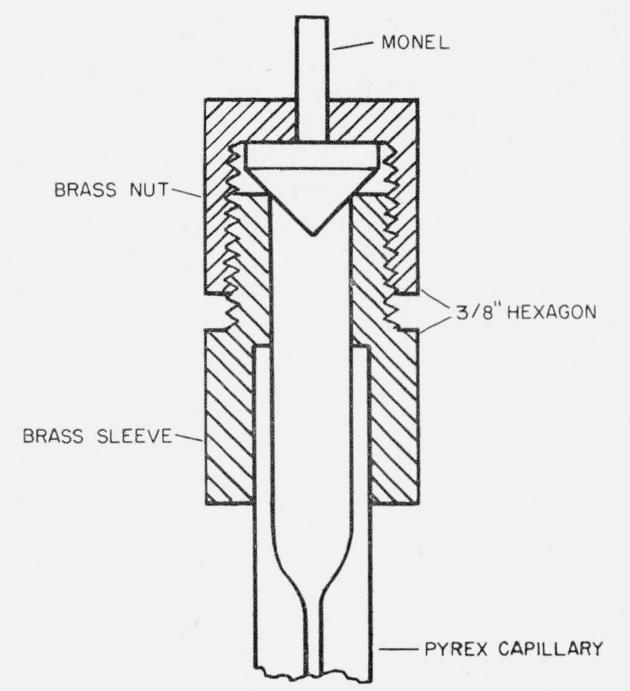

Figure 1. Schematic drawing of metal cap for pycnometer. 
ment of thermal equilibrium the caps were tightened and the pycnometers were removed from the bath, cleaned, placed in the balance case, and weighed after allowing sufficient time for the attainment of temperature equilibrium.

The pycnometers were first weighed against a tare (a similar empty pycnometer) and then against each other. With the pycnometers empty the directly measured difference in weight (corrected for air buoyancy) usually checked the difference in the weights against the tare within $0.02 \mathrm{mg}$. With the pycnometers filled the check was not always this good, but was usually within $0.05 \mathrm{mg}$ or less.

After the filled pycnometers were weighed they were immersed in the water bath to a point a little below the lower part of the metal cap. The caps themselves were heated to a temperature above that of the bath by means of electric heaters to insure against condensation of solvent in the caps. After sufficient time had elapsed to permit the system. to come to temperature equilibrium, the heaters were removed and the pycnometers were lowered so as to be completely imm.ersed in the water. After this a number of observations were made of the positions of the menisci in the capillaries relative to the reference marks etched on them.

Measurements of the heights of the menisci were facilitated by placing behind the capillaries a white Bakelite sheet with black lines about $0.3-\mathrm{mm}$ wide drawn on its surface at an angle of $45^{\circ}$ with the horizontal. This device has been described by Zeleny [6]. The menisci are sharply outlined by the apparent intersections of the black lines with their reflections in the surface of the liquid.

Measurements of the density of the sample of polyisobutene used were made to a precision of about 0.1 percent by the method of hydrostatic weighing.

\subsection{Vapor-Pressure Measurements}

Vapor pressures were determined from measurements of the difference between the vapor pressure of the purified benzene and the pressures over a weighed sample of polymer to which measured quantities of benzene had been added. The apparatus used is shown schematically in figure 2 (a). By means of the valve $\mathrm{C}$, benzene could be transferred by distillation from the buret B to the polymer in the tube $H$. The amount of benzene so transferred was determined from the change in position of the benzene meniscus in the buret and the change in position of the mercury meniscus in the left arm of the manometer $\mathrm{M}$, the diameter of which was known. In determining the mass of benzene transferred, great care was taken to insure that no liquid benzene was present in the left arm of the manometer or in the connecting tubing when the buret was being read. From the total amount of benzene transferred was subtracted the amount of vapor in the known volumes in the tube $\mathrm{H}$, the right arm of the manometer, and the connecting tubing to obtain the benzene content of the polymersolvent mixture. Benzene could also be transferred

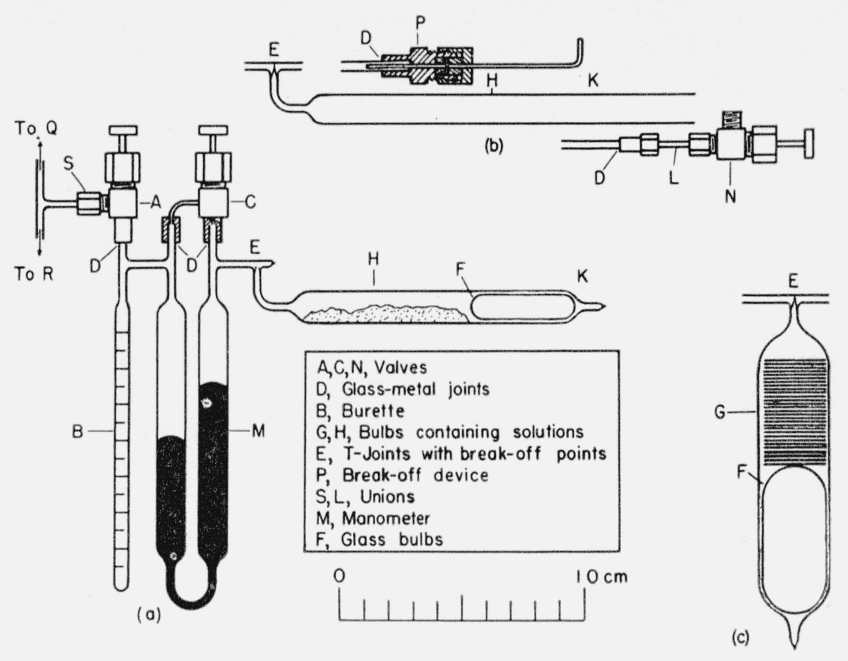

FIGURE 2. Schematic diagram of vapor-pressure apparatus.

(a) Assembled apparatus; (b) apparatus for introducing polybutadiene sample; (c) bulb for polyisobutene solutions.

from the polymer-solvent mixture to the buret B (fig. 2 (a)) by cooling the latter in liquid air and opening the valve C. Measurements of vapor pressure could thus be made both after increasing the benzene content of the mixture and after decreasing it.

The method of introducing the polybutadiene, mercury, and benzene into the apparatus will now be described. The tube $\mathrm{H}$ was initially open at one end, as shown in figure 2 (b), and was sealed at the other end to the smaller tube which contained a break-off point in the $\mathrm{T}$-joint at $\mathrm{E}$. The tube $\mathrm{H}$ was weighed empty, and again after introducing the desired amount of polybutadiene (about $1.0 \mathrm{~g}$ ). The bulb F was inserted in $\mathrm{H}$, which was then drawn down at the point $\mathrm{K}$ and sealed to a smaller tube which, in turnwas joined to the brass union $\mathrm{L}$ by means of a glassto metal soldered joint. The brass needle valve $\mathrm{N}$ was attached to the union and to a similar union leading to a vacuum system. After removal of air and benzene from the polymer sample as described in section 2.1, the valve $\mathrm{N}$ and union $\mathrm{L}$ were removed by sealing off the small tube connecting them to $\mathrm{H}$.

To introduce mercury in to the manometer, it was rotated $90^{\circ}$ clockwise from the position shown in figure 2 (a) and a small flask containing the desired amount of mercury was sealed on in place of the tube H. The system was then evacuated, the valve A was closed and disconnected from the vacuum system, and the manometer was rotated so as to allow the mercury to flow into it. With the valve $\mathrm{C}$ open, air was then admitted slowly through the valve $A$, and the flask that had contained the mercury was disconnected from the manometer.

The tube $\mathrm{H}$ was then sealed to the manometer and to the breakoff device P (fig. 2 (b)). This consisted of a brass stuffing box connected to a short piece of glass tubing by a glass-to-metal soldered joint, and having a Teflon packing through which passed a heavy phosphor bronze wire. The system was then 
evacuated through the valve $\mathrm{A}$ and a valve $\mathrm{Q}$ (not shown in figure) which was attached to the vacuum system. The connection to the vacuum system was sufficiently flexible so that the manometer could be tilted to liberate any air bubbles that might have been trapped below the mercury surfaces during the glassblowing operations. Then, with the manometer still connected to the vacuum system but with the vacuum pump shut off, the tube $H$ was opened to the manometer by sliding the phosphor bronze wire through the stuffing box $\mathrm{P}$ so as to break off the glass point at $\mathrm{E}$, and the breakoff device was removed by sealing off a short distance to the right of $\mathrm{E}$. After this, a measurement of the pressure in the vacuum system always gave less than $10^{-4} \mathrm{~mm}$ of mercury, showing that no appreciable amount of air had leaked into the system.

The buret B was then immersed in an ice bath. The valve $\mathrm{C}$ and the valve $\mathrm{Q}$ (not shown in fig. 2) on the vacuum system were closed, and the valve $R$ (not shown) on a bulb containing a supply of purified benzene was opened, allowing the benzene to distill into the buret. The buret was initially filled to a point above the highest graduation mark, the valve $R$ was closed, and the excess benzene was removed by pumping it into the vacuum system. The valve A was then closed. The buret was then immersed in an ice bath and measurements of the vapor pressure of the supercooled liquid benzene at $0^{\circ} \mathrm{C}$ were made.

The temperature of the apparatus during measurements of the difference in pressure between solvent and polymer-solvent mixtures was controlled near $26.9^{\circ} \mathrm{C}$ by the same water bath used in the density measurements. The heights of the two menisci in the manometer were measured to $0.01 \mathrm{~mm}$ by means of a Wild cathetometer, the menisci being sharply outlined by the device of Zeleny [6] described in section 3.1 .

The apparatus and procedure for measurement of the vapor pressures of polyisobutene-benzene mixtures differed from that described above only in that the sample bulb G (fig. 2 (c)) was used instead of $\mathrm{H}$. In order to promote reasonably rapid attainment of equilibrium in the polymer-solvent mixture, the polymer was spread in layers approximately $0.2 \mathrm{~mm}$ thick on brass disks spaced about $1-\mathrm{mm}$ apart in the tube $G$, the axis of which was kept vertical as shown in figure 2 (c).

\section{Results}

\subsection{Densities of Polyisobutene Solutions}

The experimental values of density at $26.900^{\circ} \mathrm{C}$ and the corresponding values of apparent specific volume of polymer, $\phi_{2}$, are given in table 1 . The values of $\phi_{2}$ are also plotted as ordinate against mass fraction of polymer in figure 3 . The values of $\phi_{2}$ were calculated from the measured densities by means of the equation

$$
\phi_{2}=\frac{1}{\rho_{1}}\left[1-\frac{\rho-\rho_{1}}{w_{2} \rho}\right],
$$

where $\rho_{1}$ and $\rho$ are the densities of solvent and solution, respectively, and $w_{2}$ is the mass fraction of polymer. The mean of the two experimental values of $\rho_{1}$ was used in this calculation.

The values of $\phi_{2}$ in the lower concentration range are much less reliable than those at the higher concentrations, since, according to eq (1), the effect on the calculated value of $\phi_{2}$ of a given error in $\rho$ (or $\left.\rho_{1}\right)$ is approximately inversely proportional to $w_{2}$. Therefore the form of the curve cannot be determined accurately at the lower concentrations, and the curve in figure 3 is dotted in this region to emphasize this fact. The curve was drawn to fit the experimental points as well as possible and to have a horizontal tangent ${ }^{4}$ at $w_{2}=0$. With one exception the points deviate from the curve as drawn by amounts corresponding to $5 \mathrm{ppm}$ or less in density. It may be noted that if the value for the density of the solvent

TABLE 1. Observed densities of polyisobutene-benzene solutions at $26.900^{\circ}$ and calculated values of apparent specific volume of polymer

\begin{tabular}{|c|c|c|}
\hline $\begin{array}{c}\text { Mass fraction } \\
\text { of polymer, } w_{2}\end{array}$ & $\begin{array}{c}\text { Density of } \\
\text { solution }\end{array}$ & $\begin{array}{c}\text { Apparent spe- } \\
\text { cific volume } \\
\text { of polymer }\end{array}$ \\
\cline { 3 - 3 } & $\begin{array}{c}\text { g/cm }{ }^{3} \\
0.87131_{4}\end{array}$ & $\mathrm{~cm}^{3} / g$ \\
\hline 0 & $.871309_{0}$ & $-.1098_{7}$ \\
0.005477 & $.871467_{5}$ & $1.1089_{6}$ \\
.005477 & $.871471_{3}$ & $1.1093_{7}$ \\
.010958 & $.871629_{2}$ & $1.1082_{1}$ \\
.019323 & $.871889_{8}$ & $1.1080_{8}$ \\
.019323 & $.871891_{7}$ & $1.1085_{2}$ \\
.030266 & $.872211_{1}$ & $1.1080_{7}$ \\
.040261 & $.872523_{1}$ & $1.1082_{4}$ \\
.040261 & $.872517_{8}$ & $1.1079_{3}$ \\
.050027 & $.872823_{2}$ & $1.1081_{4}$ \\
.050677 & $.872834_{9}$ & $1.1081_{4}$ \\
.050677 & $.872834_{6}$ & $1.107_{0}$ \\
.098313 & $.874305_{4}$ & $1.094_{7}$ \\
\hline 1.000 & $.913_{5}$ & \\
\hline
\end{tabular}

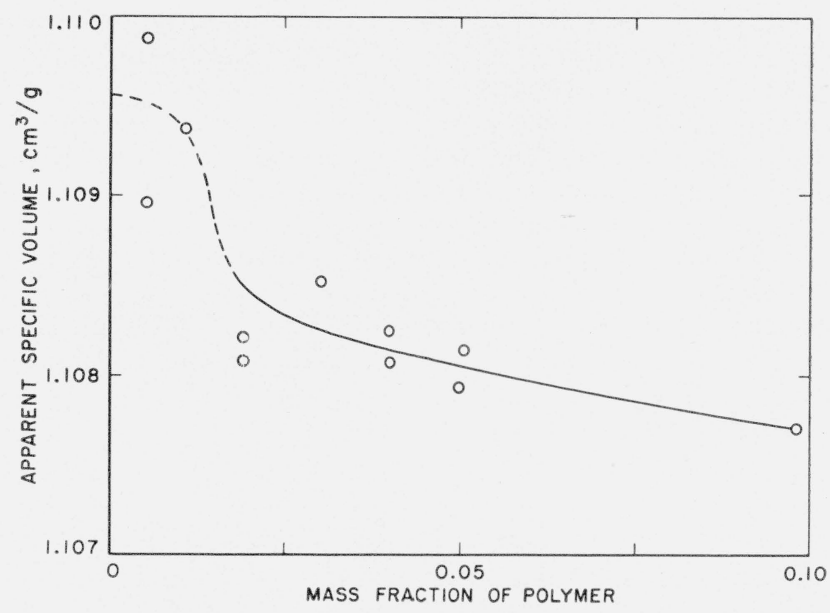

FIGURE 3. Apparent specific volume of polyisobutene in benzene at $26.90^{\circ} \mathrm{C}$.

4 This condition seems plausible from physical considerations, since in a sufficiently dilute solution the solute molecules are too far apart to interact appreciably. 
were lower by $5 \mathrm{ppm}$ the points representing the values of $\phi_{2}$ would still show a slight downward trend with increasing $w_{2}$, and could be approximately represented by a straight line going from 1.1084 at $w_{2}=0$ to 1.1076 at $w_{2}=0.10$.

A decrease in $\phi_{2}$ with increasing $w_{2}$ bas been reported by Rosen [7] for polystyrene in cyclohexane, toluene, and chloroform. The decrease in $\phi_{2}$ shown in figure 3 is much smaller in magnitude than that reported by Rosen, but the trend extends over a greater range of concentration. The density data of Streeter and Boyer [8] also lead to a decrease in apparent specific volume of polystyrene in 0 -dichlorobenzene with increasing polymer concentration in the range $w_{2}=0$ to 0.06 . Horth and Rinfret [9] have reported discontinuities in curves of density versus polymer concentration for dilute solutions of polyvinyl acetate in chlorobenzene. These discontinuities correspond to increases in apparent specific volume with increasing polymer concentration. The data in reference [4] show no change in $\phi_{2}$ with composition of solutions of polystyrene in benzene, toluene, ethylbenzene, and 2-butanone in the composition range $w_{2}=0.02$ to 0.10 .

From the curve of figure 3 and the value given in table 1 for the density of the polyisobutene sample, the change in volume when a $1-\mathrm{g}$ sample of polymer is dissolved in benzene varies from +0.013 to +0.015 $\mathrm{cm}^{3}$. As shown previously [4], a change in volume upon mixing affects such thermodynamic properties as heat of mixing, free energy of mixing, etc. It has been calculated that the change in volume when a 1-g sample of polyisobutene is dissolved in a large amount of benzene will affect the heat of mixing by about -2 calories. Since $\phi_{2}$ varies with composition as shown in figure 3 , the partial specific volume of solvent will differ from the specific volume of the pure solvent by about $2 \times 10^{-5} \mathrm{~cm}^{3} / \mathrm{g}$ at $w_{2}=0.05$. This change in volume will affect the partial molal heat of dilution by about -0.25 calorie per mole of benzene. These thermal effects resulting from volume changes are approximately equal in magnitude, but opposite in sign, to the values of heat of mixing and heat of dilution calculated by Hildebrand's equation [10], the derivation of which implies no change in volume on mixing.

\subsection{Vapor Pressures \\ a. Polybutadiene-Benzene}

The results of vapor-pressure measurements on the system polybutadiene-benzene are given in table 2 in terms of the activity $a_{1}$ of the solvent, defined as the ratio $f_{1} / f_{1}^{0}$ of the fugacity ${ }^{5}$ of the solvent in the mixture to that in the pure solvent. Also given in table 2 are the values calculated from the measured activities for the parameter $\mu$ in the Flory-Huggins [11] equation

$$
\ln a_{1}=\ln v_{1}+\left(1-\frac{1}{m}\right) v_{2}+\mu v_{2}^{2}
$$

${ }^{5}$ Pressure ratios were converted to fugacity ratios in all cases, although differ ence was usually negligible. where $m$ is the ratio of the molar volume of solute to that of solvent, and $v_{1}$ and $v_{2}$ represent volume fractions of solvent and solute respectively. In this calculation the term $1 / m$ was neglected.

The results are also shown graphically in the lower curve of figure 4 , where $a_{1}$ is plotted as ordinate against $v_{1}$ as abscissa, and in figure 5, where the calculated values of the parameter $\mu$ are plotted against $v_{1}$. Not all of the experimental points are plotted in figure 4 , since in this plot they would lie so close together as to confuse the diagram. In

TABLE 2. Results of vapor-pressure measurements in the system polybutadiene-benzene

\begin{tabular}{|c|c|c|c|}
\hline $\begin{array}{l}\text { Experi- } \\
\text { ment }\end{array}$ & $\begin{array}{l}\text { Volume } \\
\text { fraction } \\
\text { solvent, } v_{1}\end{array}$ & $\begin{array}{c}\text { Observed } \\
\text { activity } \\
\text { of solvent, } a_{1}\end{array}$ & $\mu$ \\
\hline $\begin{array}{l}1^{*} \\
2^{*} \\
3^{*} \\
4^{*} \\
5^{*}\end{array}$ & $\begin{array}{r}0.0710 \\
.1290 \\
.2033 \\
.2952 \\
.3731\end{array}$ & $\begin{array}{r}0.2392 \\
.3969 \\
.5558 \\
.6982 \\
.7901\end{array}$ & $\begin{array}{r}0.330_{9} \\
.332_{4} \\
.329_{3} \\
.314_{1} \\
.314_{0}\end{array}$ \\
\hline $\begin{array}{r}6^{*} \\
7^{*} \\
8 \\
9 \\
10\end{array}$ & $\begin{array}{l}.4581 \\
.5362 \\
.5367 \\
.4574 \\
.3722\end{array}$ & $\begin{array}{l}.8622 \\
.9106 \\
.9079 \\
.8586 \\
.7872\end{array}$ & $\begin{array}{l}.308_{2} \\
.305_{9} \\
.290_{8} \\
.296_{2} \\
.307_{5}\end{array}$ \\
\hline $\begin{array}{l}11 \\
12 \\
13 \\
14 \\
15\end{array}$ & $\begin{array}{l}.2932 \\
.2051 \\
.1167 \\
.5381 \\
.4587\end{array}$ & $\begin{array}{l}.6941 \\
.5560 \\
.3620 \\
.9074 \\
.8586\end{array}$ & $\begin{array}{l}.310_{2} \\
.320_{3} \\
.318_{9} \\
.284_{0} \\
.292_{1}\end{array}$ \\
\hline $\begin{array}{l}16 \\
17 \\
18 \\
19 \\
20\end{array}$ & $\begin{array}{l}.5369 \\
.4554 \\
.3794 \\
.2940 \\
.2051\end{array}$ & $\begin{array}{l}.9068 \\
.8567 \\
.7926 \\
.6948 \\
.5562\end{array}$ & $\begin{array}{l}.284_{8} \\
.294_{3} \\
.301_{7} \\
.309_{2} \\
.3209\end{array}$ \\
\hline $\begin{array}{l}21 \\
22^{*} \\
23^{*} \\
24^{*} \\
25^{*}\end{array}$ & $\begin{array}{l}.1256 \\
.1144 \\
.1275 \\
.2059 \\
.2940\end{array}$ & $\begin{array}{l}.3817 \\
.3572 \\
.3919 \\
.5567 \\
.6970\end{array}$ & $\begin{array}{l}.310_{1} \\
.322_{6} \\
.328_{9} \\
.318_{1} \\
.315_{4}\end{array}$ \\
\hline $\begin{array}{l}26^{*} \\
27^{*} \\
28^{*}\end{array}$ & $\begin{array}{l}.3792 \\
.4548 \\
.5349 \\
\end{array}$ & $\begin{array}{l}.7963 \\
.8600 \\
.9103 \\
\end{array}$ & $\begin{array}{l}.314_{2} \\
.310_{9} \\
.307_{9}\end{array}$ \\
\hline
\end{tabular}

*Experiments marked with an asterisk were made after increasing the benzene content of the mixture; all others were made after decreasing the benzene content.

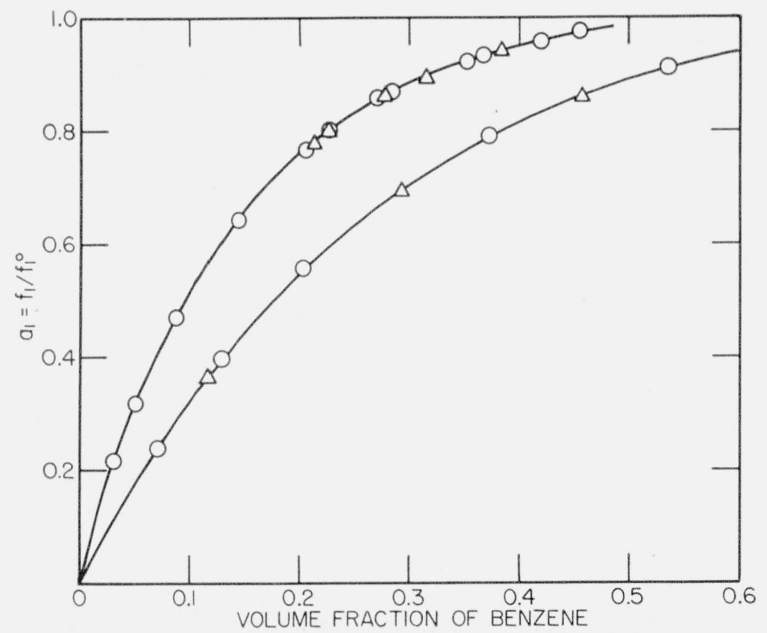

FIGURE 4. Activity of benzene in mixtures with polyisobutene (upper curve) and with polybutadiene (lower curve). 


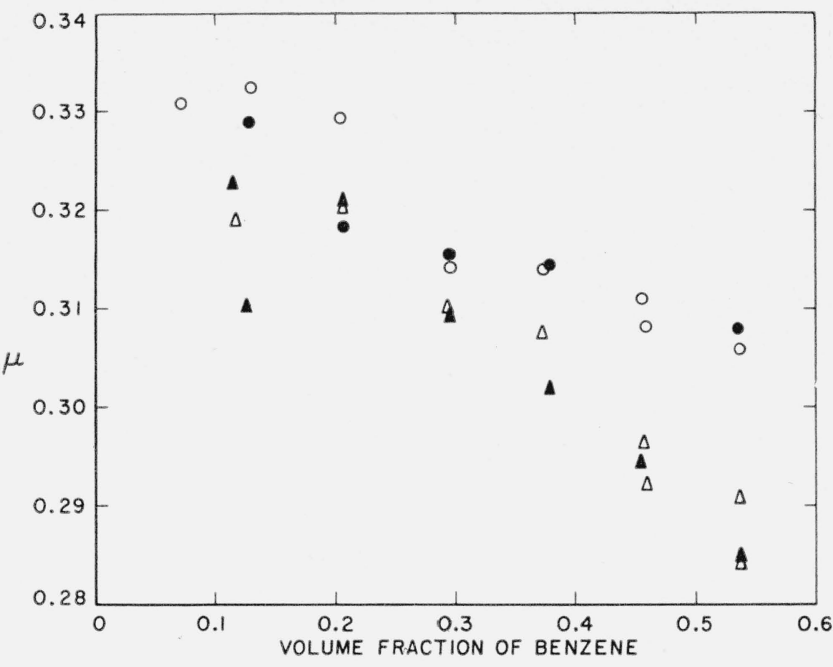

FIGURE 5. Values of the parameter $\mu$ for the system polybutadiene-benzene.

figure 5 the circles represent measurements made after increasing the benzene content of the mixture, while the triangles represent measurements taken after decreasing the benzene content. The open and solid symbols represent two different series of measurements.

After completing a preliminary series of measurements it was observed that when the benzene in the buret was frozen in liquid air, gas bubbles were liberated indicating the presence of permanent gas in the system. In view of the preliminary treatment of the polymer to remove dissolved gases it was at first thought that the presence of gas in the system indicated a leak in the apparatus. However, sensitive tests failed to show any such leak. It was finally concluded that the preliminary treatment of the polymer did not remove permanent gases completely. Therefore, before proceeding with the measurements, a series of experiments was performed in which benzene was added to and then removed from the polymer. It was found that each time this was done a small amount of gas was present in the benzene, and a small amount of benzene was pumped off to remove the gas. The amount of gas removed per experiment could not be determined quantitatively, but appeared to decrease as the number of experiments increased. After it was judged that only a negligible amount of gas remained, the vapor-pressure measurements were resumed. After the first series of measurements indicated by the open symbols in figure 5, a small amount of gas was found to be present in the system. The benzene was then transferred from the buret to the mixture and left for about a month, after which a second series of measurements of vapor pressure was made, the results of which are represented by the solid symbols in figure 5. Although the amount of gas in the system appeared to be considerably greater after the second series of measurements than after the first, it will be seen from figure 5 that there was little if any systematic difference between results obtained

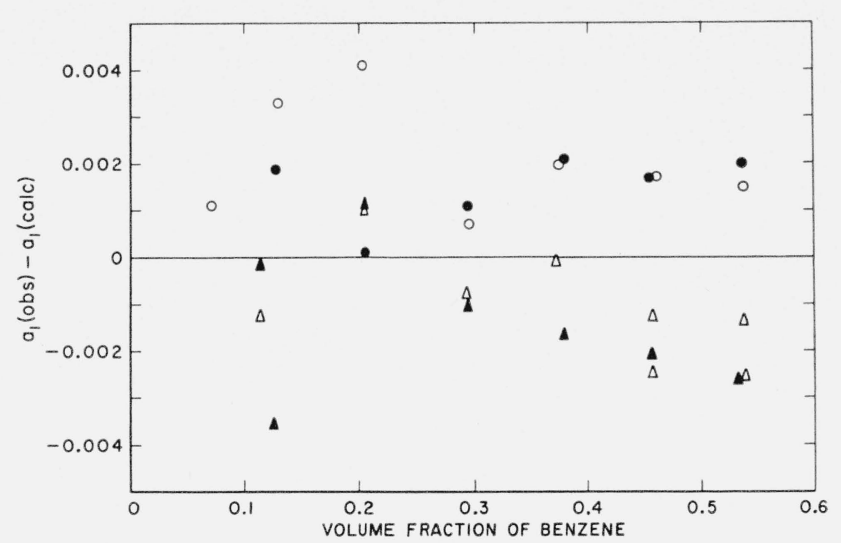

FIGURE 6. Deviations of observed activities in benzene-polybutadiene system from equation (2).

under the same conditions in the two series. This indicates that the amount of air present was not sufficient to affect the measured vapor pressures appreciably, and that the systematic difference between results obtained after increasing and after decreasing the benzene content of the mixture is due to hysteresis in the system polybutadiene-benzene.

The variation of $\mu$ with $v_{1}$ (fig. 5) shows that the vapor pressures in this system cannot be represented within the precision of the measurements by the Flory-Huggins equation. They can be represented, however, by adding a term in $v_{2}^{3}$ to this equation. Figure 6 shows deviations of the measured values of $a_{1}$ from

$$
\ln a_{1}=\ln v_{1}+v_{2}+0.27 v_{2}^{2}+0.06 v_{2}^{3} .
$$

\section{b. Polyisobutene-Benzene}

The results of the vapor-pressure measurements in the system polyisobutene-benzene are given in table 3, and are shown graphically by the upper

TABLE 3. Results of vapor-pressure measurements in the system polyisobutene-benzene

\begin{tabular}{|c|c|c|c|}
\hline $\begin{array}{l}\text { Experi- } \\
\text { ment }\end{array}$ & $\begin{array}{l}\text { Volume frac- } \\
\text { tion of sol- } \\
\text { vent, } v_{1}\end{array}$ & $\begin{array}{l}\text { Activity of } \\
\text { solvent, } a_{1}\end{array}$ & $\mu$ \\
\hline $\begin{array}{l}1^{*} \\
2^{*} \\
3^{*} \\
4^{*} \\
5^{*}\end{array}$ & $\begin{array}{r}0.0310 \\
.0509 \\
.0879 \\
.1439 \\
.2053\end{array}$ & $\begin{array}{r}0.2162 \\
.3159 \\
.4684 \\
.6410 \\
.7664\end{array}$ & $\begin{array}{r}1.037_{5} \\
.975_{5} \\
.916_{1} \\
.871_{5} \\
.9290\end{array}$ \\
\hline $\begin{array}{r}6^{*} \\
7^{*} \\
8^{*} \\
9 \\
10\end{array}$ & $\begin{array}{l}.2845 \\
.3528 \\
.4204 \\
.3844 \\
.3161\end{array}$ & $\begin{array}{l}.8689 \\
.9229 \\
.9557 \\
.9398 \\
.8937\end{array}$ & $\begin{array}{l}.784_{9} \\
.752_{4} \\
.721_{0} \\
.7364 \\
.761_{4}\end{array}$ \\
\hline $\begin{array}{l}11 \\
12^{*} \\
13^{*} \\
14^{*} \\
15\end{array}$ & $\begin{array}{l}.2283 \\
.2283 \\
.2858 \\
.3683 \\
.2787\end{array}$ & $\begin{array}{l}.7989 \\
.8021 \\
.8717 \\
.9325 \\
.8610\end{array}$ & $\begin{array}{l}.808 \\
.815 \\
.787 \\
.746 \\
.783\end{array}$ \\
\hline $\begin{array}{l}16^{*} \\
17 \\
18^{*} \\
19^{*} \\
20^{*}\end{array}$ & $\begin{array}{l}.2787 \\
.2140 \\
.2140 \\
.2719 \\
.3563\end{array}$ & $\begin{array}{l}.8634 \\
.7766 \\
.7806 \\
.8586 \\
.9306\end{array}$ & $\begin{array}{l}.788_{4} \\
.815_{5} \\
.823_{7} \\
.797 \\
.765\end{array}$ \\
\hline $\begin{array}{l}21^{*} \\
22\end{array}$ & $\begin{array}{l}.4567 \\
.4230\end{array}$ & $\begin{array}{l}.9745 \\
.9600\end{array}$ & $\begin{array}{l}.7290 \\
.730 s\end{array}$ \\
\hline
\end{tabular}

* Experiments marked with an asterisk were made after increasing the benzene content of the mixture; all others were made after decreasing the benzene content. 


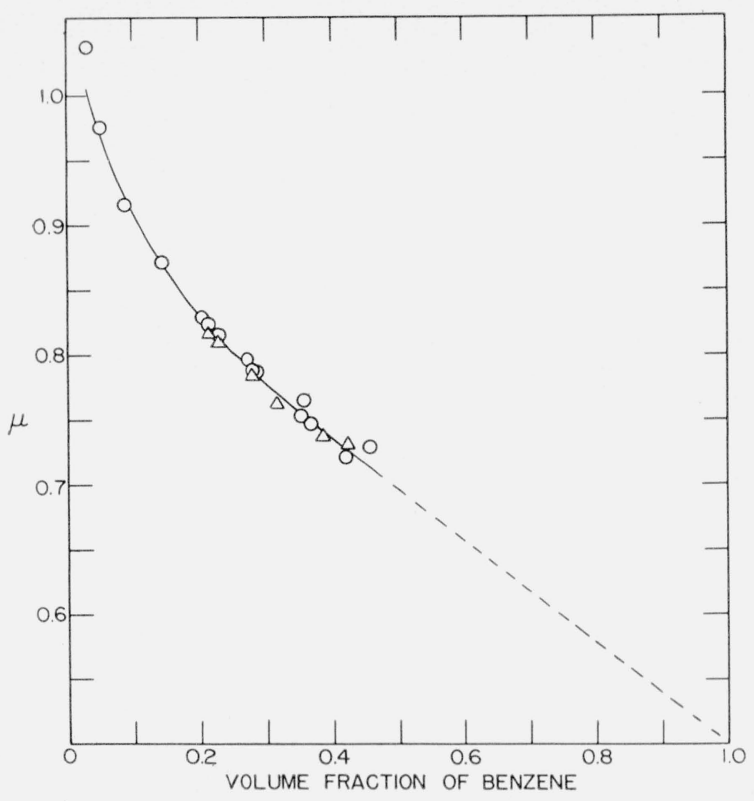

FIgURE 7. Values of the parameter $\mu$ for the system polyisobutene-benzene.

curve in figure 4 . The values of the parameter $\mu$ given in table 3 are shown graphically in figure 7 . The circles in this figure represent observations made after increasing the benzene content of the mixture, while the triangles represent observations made after decreasing the benzene content. The value of $m$ used in this calculation was taken as the ratio of the molal volumes of polymer and solvent, namely 928. The dotted straight line in figure 7 which is tangent to the lower end of the experimental curve passes through the value $\mu=0.5$ at $v_{1}=1$. This value of $\mu$ has been reported [12] for dilute solutions of polyisobutene in benzene.

The same difficulties in removing air from the mixture were encountered with this system as with the system polybutadiene-benzene. It is not believed, however, that air in the system contributed appreciably to the hysteresis shown by the usually systematic deviations of circles and triangles from the curve in figure 7 .

It is seen by comparison of figures 5 and 7 that the deviations from the Flory-Huggins equation and the variation of the parameter $\mu$ with composition are much greater for the polyisobutene-benzene system than for the polybutadiene-benzene system. It is not possible to represent the data on the polyisobutene-benzene system by as simple an equation as (2), but these data can be represented by

$$
\ln a_{1}=\ln v_{1}+\left(1-\frac{1}{m}\right) v_{2}+0.5 v_{2}^{2}+0.388 v_{2}^{3}+0.184 v_{2}^{12} .
$$

Deviations of the experimental points from this equation are shown in figure 8 . For this system the agreement between observations made under comparable conditions is generally less satisfactory, and the deviations of experimental from calculated

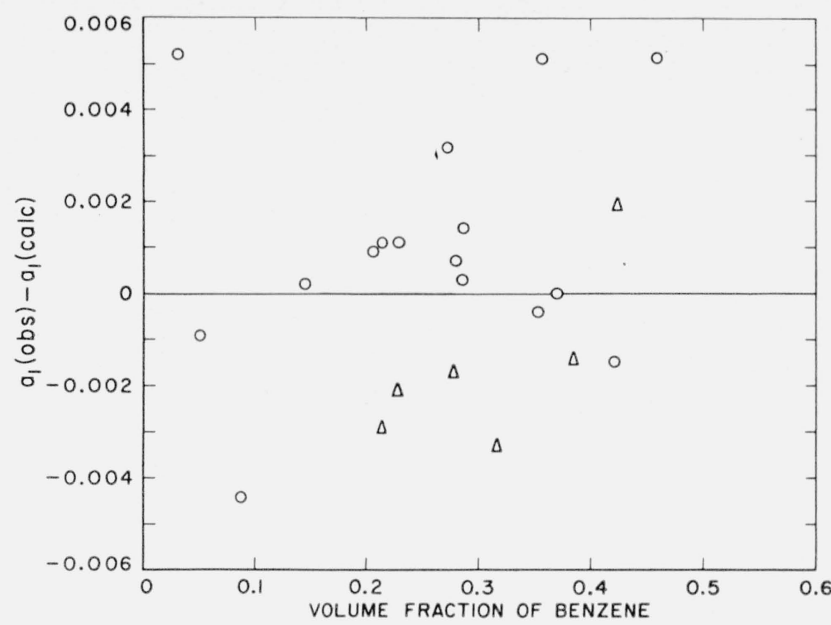

Figure 8. Deviations of observed activities in the system polyisobutene-benzene from equation (3).

activities are somewhat larger than for the system polybutadiene-benzene. This may be related to the fact that the time required for the attainment of equilibrium in the polyisobutene solutions is much greater than for the polybutadiene solutions, and therefore departures from equilibrium resulting from unavoidable fluctuations in temperature are likely to be larger in the polyisobutene solutions.

The vapor-pressure data reported here for the system polyisobutene-benzene at $26.9^{\circ} \mathrm{C}$ are in fair agreement with results at $25^{\circ} \mathrm{C}$ reported by Bawn and Patel [13] for solutions in benzene of a polyisobutene of molecular weight 45,000 . Their curve representing values of the paramenter $\mu$ is somewhat lower at low values of $v_{1}$ than that shown in figure 7 , and the two curves intersect at about $v_{1}=0.5$. The difference between the two curves at $v_{1}=0.1$ corresponds to about 3 percent in $a_{1}$.

\section{References}

[1] R. A. Nelson, R. S. Jessup, and D. E. Roberts, J. Research NBS 48, 275 (1952) RP2313.

[2] F. W. Schwab and E. Wichers, J. Research NBS 25, 747 (1940) RP1351.

[3] Selected values of properties of hydrocarbons, Am. Petroleum Inst. Research Proj. 44, Carnegie Inst. Technology, (a) table 5a (1950); (b) table 5k (1954).

[4] M. Griffel, R. S. Jessup, J. A. Cogliano, and Rita P. Park, J. Research NBS 52, 217 (1954) RP2492.

[5] W. Heller and A. C. Thompson, J. Colloid Sci. 6, 57 (1951).

[6] J. Zeleny, Rev. Sci. Instr. 7, 289 (1936).

[7] B. Rosen, J. Polymer Sci. 1\%, 559 (1955).

[8] D. J. Streeter and R. F. Boyer, Ind. Eng. Chem. 43, 1790 (1951)

[9] A. Horth and M. Rinfret, J. Am. Chem. Soc. $\mathbf{y} \%, 503$ (1955).

[10] J. H. Hildebrand and R. L. Scott, Solubility of nonelectrolytes, chap. VII, eq (34) and (35) (Rheinhold Publishing Co., Inc., New York, N. Y., 1950).

[11] See, for example, P. J. Flory, Principles of polymer chemistry, (Cornell Univ. Press, Ithaca, N. Y., 1953); M. L. Huggins, J. Polymer Sci. 16, 209 (1955).

[12] Reference 10 p. 362, based on data by P. J. Flory, J. Am. Chem. Soc. 65, 375 (1943).

[13] C. E. H. Bawn and R. D. Patel, Trans. Faraday Soc. 52, 1665 (1956).

Washington, April 22, 1957. 